\title{
PRINCIPAL HOMOGENEOUS SPACES OVER HENSEL RINGS
}

\author{
ROSARIO STRANO
}

ABSTRACT. We prove that if $(A, \underline{a})$ is a Hensel couple and $G$ is an affine, smooth group scheme over $A$ then $H_{\mathrm{et}}^{1}(A, G)=H_{\mathrm{et}}^{1}(A / \underline{a}, G / \underline{a} G)$.

Introduction. In this paper we prove the following

THEOREM 1. Let $(A, \underline{a})$ be a Hensel couple and let $G$ be an affine, smooth group scheme over $A$, i.e. a functor $G:(A$-algebras $) \rightarrow$ (groups) which is represented by a smooth A-algebra (also denoted $G$ ). Then the canonical map $H_{\mathrm{et}}^{1}(A, G) \rightarrow$ $H_{\mathrm{et}}^{1}(A / \underline{a}, G / \underline{a} G)$ is bijective.

This theorem generalizes a result known when $A$ is local and $\underline{a}$ is the maximal ideal (see [4, Theorem 11.7]).

The injectivity of the above map was proved in [8]. We also use the main result of [3].

Recall that $H_{\mathrm{et}}^{1}(A, G)$ classifies the isomorphism classes of principal homogeneous spaces in the étale topology over $A$ under $G$ (PHS for short, "torseurs" in French terminology).

In $\S \S 1$ and 2 we give some preliminaries. In $\S 3$ we prove Theorem 1 in the case when $A$ is an AIC ring, and we conclude the proof in $\$ 4$, where some corollaries are also given.

As for the notion of Hensel couple, see [2]; all the notions about group schemes and cohomology can be found in [1 and 6].

1. In this section we give the definition and some properties of absolutely integrally closed rings that we will use later (see also [3, 2.B]).

A ring $A$ is said to be absolutely integrally closed (AIC for short) if it satisfies one of the following equivalent conditions:

(a) every monic polynomial in $A[X]$ has a root in $A$;

(b) every monic polynomial in $A[X]$ splits into a product of linear factors.

Proposition 1. The following properties hold:

(1) Every ring of fractions and every quotient of an AIC ring is AIC.

(2) $A$ local $A I C$ ring is strict henselian.

(3) If $A$ is an $A I C$ ring and $\underline{a} \subset A$ is an ideal, then the henselization ${ }^{h}(A, \underline{a})$ is $A I C$.

Received by the editors January 20, 1982.

1980 Mathematics Subject Classification. Primary 13J15, 14F20; Secondary 14L15.

'This paper was done within the Group for Algebra and Geometry (GNSAGA) of the Italian National Research Council (CNR). 
Proof. (1) and (2) are easy. (3) By [2, Theorem 6.1], ${ }^{h}(A, \underline{a})$ is a direct limit of $N$-extensions of $(A, \underline{a})$; hence we reduce to the case of $B=A[x]_{1+(a . x) A \mid x]}$ where $A[x]=A[X] /(f)$ and $f$ is an $N$-polynomial, i.e. $f=X^{n}+a_{n-1} X^{n-1}+\cdots+a_{1} X$ $+a_{0}$ with $a_{0} \in \underline{a}, a_{1}$ invertible modulo $\underline{a}$. We write $f=\left(X-b_{1}\right) \cdots\left(X-b_{n}\right)$, $b_{i} \in A$, and show that $B=\prod_{i=1}^{n} A_{1+(a, b,)}$. In fact for every $i=1, \ldots, n$ if we put $f=\left(X-b_{i}\right) g_{i}(X)$, we have $f^{\prime}(X)=\bar{g}_{i}(X)+\left(X-b_{i}\right) g_{i}^{\prime}(X)$; but the image $f^{\prime}(x)$ of $f^{\prime}(X)$ in $B$ is invertible since $f^{\prime}(x) \in 1+(\underline{a}, x) A[x]$.

Given any ring $A$ we can construct a ring $A^{*}$ as follows: let $\left\{f_{i}\right\}_{i \in I}$ be the set of monic polynomials in $A[X]$ and put $A_{i}=A[X] /\left(f_{i}\right)$; define $A_{1}=\otimes_{i \in I} A_{i}$ and, inductively, $A_{n}=\left(A_{n-1}\right)_{1}$; finally let $A^{*}=\lim A_{n}$.

It is easy to see that the ring $A^{*}$ is an AIC ring, faithfully flat and integral over $A$, and it is the direct limit of $A$-algebras which are free with finite rank as $A$-modules.

2. In this section we deduce from the main result of [3] a method of descent for modules and algebras.

Let $(A, \underline{a})$ be an $H$-couple. For every $f \in A$ we denote by ${ }^{h} A$ the henselization of $A_{f}$ with respect to $\underline{a} A_{f}$. Let $f_{1}, \ldots, f_{n} \in A$ such that $\left(f_{1}, \ldots, f_{n}\right)=A$. In [3, Theorem 1.11] we proved that if $M_{i}$ are ${ }^{h} A_{f_{i}}$-modules with isomorphisms

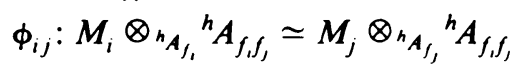

and the $\phi_{i j}$ 's satisfy the cocycle condition $\phi_{i j} \circ \phi_{j k}=\phi_{i k}$ on ${ }^{h} A_{f_{1} f_{f_{k}}}$, then there is a unique $A$-module $M$ such that $M \otimes_{A}{ }^{h} A_{f_{i}} \simeq M_{i}, i=1, \ldots, n$. This easily particular we apply this "henselian descent" to the following case. Let $G$ be an affine group scheme of finite presentation over $A$. Recall that a principal homogeneous space for the étale topology over $A$ under $G$ is a $G$-space, i.e. an $A$-algebra $H$ together with a $G$-action (see [1, II, 1.3.1]), such that $H \otimes_{A} S \simeq G \otimes_{A} S$ as $G \otimes_{A} S$-spaces over $S$ for some faithfully flat étale homomorphism $A \rightarrow S$.

Now suppose we have PHS's $H_{i}$ over ${ }^{h} A_{f_{i}}$ and isomorphisms

$$
\phi_{i j}: H_{i} \otimes_{h_{A} f_{i}}{ }^{h} A_{f_{i} f_{j}} \simeq H_{j} \otimes_{h_{A_{f j}}}{ }^{h} A_{f_{i} f_{j}}
$$

as $G \otimes_{A}{ }^{h} A_{f_{1} f_{j}}$-spaces over ${ }^{h} A_{f_{i} f_{j}}$, such that the $\phi_{i j}$ 's satisfy the cocycle condition over ${ }^{h} A_{f_{1}, f_{k}}$; then there is a unique PHS $H$ over $A$ such that $H \otimes{ }_{A}{ }^{h} A_{f_{i}} \simeq H_{i}$ as $G \otimes_{A}{ }^{h} A_{f_{i}^{-}}$ spaces over ${ }^{h} A_{f_{i}}$. In fact from the above remark we can descend the algebras $H_{i}$ and the $G \otimes_{A}{ }^{h} A_{f_{1}}$-action and obtain a $G$-space $H$ over $A$; moreover $H$ is of finite presentation over $A$ since the $H_{i}$ 's are of finite presentation over ${ }^{h} A_{f_{i}}$; and, since $H \otimes_{A} S_{i} \simeq G \otimes_{A} S_{i}$ as $G \otimes_{A} S_{i}$-spaces over $S_{i}$ for faithfully flat étale ${ }^{h} A_{f_{i}}$-algebras $S_{i}$, we easily see that there exists a faithfully flat étale $A$-algebra $S$ such that $H \otimes_{A} S \simeq$ $G \otimes_{A} S$ as $G \otimes_{A} S$-spaces over $S$. Thus $H$ is a PHS over $\ddot{A}$ under $G$.

3. In this section we prove Theorem 1 in the case when $A$ is an AIC ring. First of all we recall some known facts about cohomology.

Proposition 2. Let $G$ be an affine smooth group scheme over $A$; then

(a) $H_{\mathrm{et}}^{1}(A, G) \simeq H_{\mathrm{fp}}^{1}(A, G)$, where $\mathrm{fp}$ denotes the faithfully flat finite presentation topology, and

(b) if $(A, \underline{a})$ is an $H$-couple, the canonical map $H_{\mathrm{et}}^{1}(A, G) \rightarrow H_{\mathrm{et}}^{1}(A / \underline{a}, G / \underline{a} G)$ is injective. 
Proof. (a) See [4, Corollary 11.9].

(b) See [8, I, Theorem 2 and II, Proposition 2].

THEOREM 2. Let $(A, \underline{a})$ be an $H$-couple and let $G$ be an affine smooth group scheme over $A$. Suppose $A$ is an $A I C$ ring. Then

$$
H_{\mathrm{et}}^{\mathrm{l}}(A, G) \simeq H_{\mathrm{et}}^{1}(A / \underline{a}, G / \underline{a} G) .
$$

Proof. We have to prove only surjectivity. Let $\bar{H}$ be a PHS over $\bar{A}=A / a$, and let $I \subset A$ be the set of elements $f \in A$ such that there exists a PHS $H(f)$ over ${ }^{h} A_{f}$ for which $H(f) / \underline{a} H(f) \simeq \bar{H}_{f}$ as $G_{f} / \underline{a} G_{f}$ spaces. We want to prove that $I=A$. Let $p \in \operatorname{Spec} A$; since $\bar{H} \otimes_{A} A_{p}$ is trivial by Proposition 1(2), there exists $f \notin p$ such that $\bar{H}_{f}$ is trivial; hence $\bar{H}_{f}$ can be lifted to $G \otimes_{A}{ }^{h} A_{f}$. It remains to prove that $\bar{I}$ is an ideal of $A$. Let $f, g \in I$; replacing $A$ with ${ }^{h} A_{s}, s=f+g$, we can suppose $(f, g)=A$. So we have PHS's $H(f), H(g)$ over ${ }^{h} A_{f},{ }^{h} A_{g}$, respectively, such that $H(f) / \underline{a} H(f) \simeq \bar{H}_{f}$,

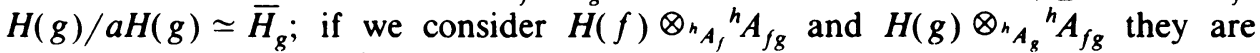
isomorphic PHS's over ${ }^{h} A_{f g}$ because of Proposition 2(b). Then by the remark in $\S 2$ there exists a PHS $H$ over $A$ such that $H / \underline{a} H \simeq \bar{H}$.

4. In this section we conclude the proof of Theorem 1 and deduce some corollaries.

Proof OF THEOREM 1 . We have to prove the surjectivity of the map $H_{\mathrm{et}}^{1}(A, G) \rightarrow$ $H_{\mathrm{et}}^{1}(\bar{A}, \bar{G})$, where $\bar{A}=A / \underline{a}, \bar{G}=G / \underline{a} G$. Let $\bar{H}$ be a PHS over $\bar{A}$ under $\bar{G}$ and consider the ring $A^{*}$ defined in $\S 1 ; \bar{H} \otimes_{\bar{A}}^{-} A^{*}$ is a PHS over $A^{*} / \underline{a} A^{*}$ under $G \otimes_{A} A^{*} / \underline{a} A^{*}$ and hence by Theorem 2 it can be lifted to a PHS $H^{*}$ over $A^{*}$ such that $H^{*} \otimes_{A} \bar{A} \simeq \bar{H} \otimes_{A} A^{*}$. Since $A^{*}$ is the limit of $A$-algebras which are free with finite rank as $A$-modules, we can find such an algebra $A^{\prime}$ and a PHS $H^{\prime}$ over $A^{\prime}$ such that $H^{\prime} \otimes_{A} \bar{A} \simeq \bar{H} \otimes_{\bar{A}} A^{\prime}$.

Now we want to descend $H^{\prime}$ to a PHS $H$ over $A$ such that $H / \underline{a} H \simeq \bar{H}$. In order to do this consider the two extensions $H_{1}, H_{2}$ of $H^{\prime}$ to $A^{\prime} \otimes_{A} A^{\prime}$ and the three extensions $H_{12}, H_{23}, H_{13}$ of $H^{\prime}$ to $A^{\prime} \otimes_{A} A^{\prime} \otimes_{A} A^{\prime}$. Let us consider the three functors $G_{1}, G_{2}, G_{3}$ : ( $A$-algebras) $\rightarrow$ (sets) defined as follows: for every $A$-algebra $B$,

$$
\begin{array}{ll}
G_{1}(B)=\operatorname{Isom}\left(H^{\prime} \otimes_{A} B, H^{\prime} \otimes_{A} B\right) & \text { as } G \otimes_{A} A^{\prime} \otimes_{A} B \text {-spaces over } A^{\prime} \otimes_{A} B, \\
G_{2}(B)=\operatorname{Isom}\left(H_{1} \otimes_{A} B, H_{2} \otimes_{A} B\right) & \text { as } G \otimes_{A} A^{\prime} \otimes_{A} A^{\prime} \otimes_{A} B \text {-spaces } \\
& \text { over } A^{\prime} \otimes_{A} A^{\prime} \otimes_{A} B, \\
G_{3}(B)=\operatorname{Isom}\left(H_{23} \otimes_{A} B, H_{12} \otimes_{A} B\right) & \text { as } G \otimes_{A} A^{\prime} \otimes_{A} A^{\prime} \otimes_{A} A^{\prime} \otimes_{A} B \text {-spaces } \\
& \text { over } A^{\prime} \otimes_{A} A^{\prime} \otimes_{A} A^{\prime} \otimes_{A} B .
\end{array}
$$

First we prove that the $G_{i}$ 's are representable by smooth $A$-algebras. In fact we consider the functor $G_{1}^{\prime}:\left(A^{\prime}\right.$-algebras) $\rightarrow$ (sets) defined by

$$
G_{1}^{\prime}(C)=\operatorname{Isom}\left(H^{\prime} \otimes_{A^{\prime}} C, H^{\prime} \otimes_{A^{\prime}} C\right)
$$

as $G \otimes_{A} C$-spaces over $C$, for every $A^{\prime}$-algebra $C$; we have $G_{1}=\prod_{A^{\prime} / A} G_{1}^{\prime}$, i.e. $G_{1}$ is the Weil restriction of $G_{1}^{\prime}$ from $A^{\prime}$ to $A$ (see $\left.[1, I, 1.6 .6]\right)$. Hence by $[1, I, 4.4 .8]$ in order to prove that $G_{1}$ is representable by a smooth $A$-algebra it is enough to prove that $G_{1}^{\prime}$ is representable by a smooth $A^{\prime}$-algebra. 
Since $H^{\prime}$ is split by a faithfully flat $A^{\prime}$-algebra $S$, the extended functor $G_{1}^{\prime} \otimes_{A^{\prime}} S$ : ( $S$-algebras) $\rightarrow$ (sets) is isomorphic to $G \otimes_{A} S$ : in fact for every $S$-algebra $D$ we have

$$
\begin{aligned}
\left(G_{1}^{\prime} \otimes_{A^{\prime}} S\right)(D) & =\operatorname{Isom}_{G \otimes_{A} D \text {-spaces over } D}\left(H^{\prime} \otimes_{A^{\prime}} D, H^{\prime} \otimes_{A^{\prime}} D\right) \\
& \simeq \operatorname{Isom}_{G \otimes_{A} D \text {-spaces over } D}\left(G \otimes_{A} D, G \otimes_{A} D\right)=\left(G \otimes_{A} S\right)(D) .
\end{aligned}
$$

Since $G \otimes_{A} S$ is representable by a smooth $S$-algebra, by [8, I, Proposition 1], $G_{1}^{\prime}$ is representable by a smooth $A^{\prime}$-algebra. In the same way we prove that $G_{2}, G_{3}$ are representable by smooth $A$-algebras.

Now we consider the functor $K:(A$-algebras) $\rightarrow$ (sets) defined as the kernel

$$
K(B)=\operatorname{Ker}\left(G_{2}(B) \underset{\theta_{1} \cdot \theta_{3}}{\stackrel{\theta_{2}}{\Rightarrow}} G_{3}(B)\right)
$$

for every $A$-algebra $B$, where $\theta_{i}$ are the maps induced by the homomorphisms $\varepsilon_{i}$ : $A^{\prime} \otimes_{A} A^{\prime} \rightarrow A^{\prime} \otimes_{A} A^{\prime} \otimes_{A} A^{\prime}$; by [8, I, Proposition 2], $K$ is representable by an $A$-algebra of finite presentation.

If we prove that $K$ is smooth, by [5, Theorem 1.8] we have that the map $K(A) \rightarrow K(\bar{A})$ is surjective, and hence we can lift any descent datum in $K(\bar{A})$ which corresponds to the $\bar{G}$-space $\bar{H}$ to a descent datum in $K(A)$ and obtain a $G$-space $H$ over $A$ which is a PHS over $A$ by Proposition 2 (a) since it is split by a faithfully flat $A$-algebra of finite presentation $A \rightarrow A^{\prime} \rightarrow S$. It remains to prove that $K$ is smooth, i.e. for every $A$-algebra $B$ and nilpotent ideal $I \subset B$ the map $K(B) \rightarrow K(B / I)$ is surjective. In fact let $\tilde{\alpha} \in K(B / I)$ : $\tilde{\alpha}$ defines a $G \otimes_{A} B / I$-space $\tilde{H}$ over $B / I$ which is a PHS since it is split by a faithfully flat $B / I$-algebra of finite presentation $B / I \rightarrow A^{\prime} \otimes_{A} B / I \rightarrow S \otimes_{A} B / I$. From the bijection

$$
H_{\mathrm{et}}^{1}\left(B, G \otimes_{A} B\right) \simeq H_{\mathrm{et}}^{1}\left(B / I, G \otimes_{A} B / I\right)
$$

(see [8, I, Theorem 4 and II, Proposition 2]) it follows that $\tilde{H}$ can be lifted to a PHS over $B$. From this, using the fact that $G_{1}$ is smooth, we can see easily that there is an $\alpha \in K(B)$ whose image in $K(B / I)$ is $\tilde{\alpha}$.

As an application of Theorem 1 we deduce some properties of $H$-couples, some of them already known (see [5 and 7]).

COROllary 1. Let $(A, \underline{a})$ be an $H$-couple, and

(a) let $P_{n}(A)$ be the set of isomorphism classes of projective A-modules with rank $n$, then $P_{n}(A) \simeq P_{n}(A / \underline{a})$; in particular $\operatorname{Pic}(A) \simeq \operatorname{Pic}(A / \underline{a})$;

(b) let $\mathrm{Az}_{n}(A)$ be the set of isomorphism classes of Azumaya A-algebras with rank $n^{2} ;$ then $\mathrm{Az}_{n}(A) \simeq \mathrm{Az}_{n}(A / \underline{a})$;

(c) let $\mathrm{Et}_{n}(A)$ be the set of isomorphism classes of étale finite A-algebras with rank $n$; then $\mathrm{Et}_{n}(A) \simeq \mathrm{Et}_{n}(A / \underline{a})$;

(d) let $Q_{2 n}$ be the set of isomorphism classes of quadratic $A$-modules of mark $\left(A^{n} \times A^{n}, q\right), \quad q(x, y)=x_{1} y_{1}+\cdots+x_{n} y_{n} \quad\left(\right.$ see $\left[1\right.$, III, 5.2.3]); then $Q_{2 n}(A) \simeq$ $Q_{2 n}(A / \underline{a})$.

Proof. In fact all the sets considered above are of the form $H_{\mathrm{et}}^{1}(A, G)$ with $G$ an affine smooth group scheme over $A$, precisely:

(a) $G=\mathrm{Gl}_{n}$, the general linear group of order $n$; 
(b) $G=\mathrm{Pgl}_{n}$, the general projective linear group of order $n$;

(c) $G=S_{n}$, the symmetric group of order $n$;

(d) $G=D_{2 n}$, the orthogonal group of order $2 n$.

COROllary 2. Let $(A, \underline{a})$ be an $H$-couple, with $A$-algebra over an algebraically closed field $k$, and let $G$ be an affine algebraic group over $k$. Then

$$
H_{\mathrm{et}}^{1}\left(A, G \otimes_{k} A\right) \simeq H_{\mathrm{et}}^{1}\left(A / \underline{a}, G \otimes_{k} A / \underline{a}\right) .
$$

Proof. In fact $G$ is smooth.

Corollary 3. Let $(A, \underline{a})$ be an $H$-couple and suppose that $n$ is a unit in $A$. Let $\mu_{n}$ be the group of n-roots of unity, i.e. for every $A$-algebra $B, \mu_{n}(B)=\left\{b \in B \mid b^{n}=1\right\}$. Then

$$
H_{\mathrm{et}}^{1}\left(A, \mu_{n}\right) \simeq H_{\mathrm{et}}^{1}\left(A / \underline{a}, \mu_{n} \otimes_{A} A / \underline{a}\right) .
$$

Proof. In the above hypothesis $\mu_{n}$ is smooth.

\section{REFERENCES}

1. M. Demazure and P. Gabriel, Groupes algébriques, North-Holland, Amsterdam, 1970.

2. S. Greco, Henselization of a ring with respect to an ideal, Trans. Amer. Math. Soc. 144 (1969), 43-65.

3. S. Greco and R. Strano, Quasi-coherent sheaves over affine Hensel schemes, Trans. Amer. Math. Soc. 268 (1981), 445-465.

4. A. Grothendieck, Le groupe de Brauer. III: Dix exposés sur la cohomologie des schèmas, NorthHolland, Amsterdam, 1968.

5. L. Gruson, Une propriété des couples henséliennes, Colloq. Algèbre Comm., Exp. 10, Rennes, 1972.

6. J. S. Milne, Etale cohomology, Math. Notes Ser., Princeton Univ. Press, Princeton, N. J., 1980.

7. R. Strano, Azumaya algebras over Hensel rings, Pacific J. Math. 61 (1975), 295-303.

8. , Cohomology of an affine group scheme over a Hensel ring, J. Algebra 47 (1977), 138-153.

Seminario Matematico, I95125 Catania, Italy 